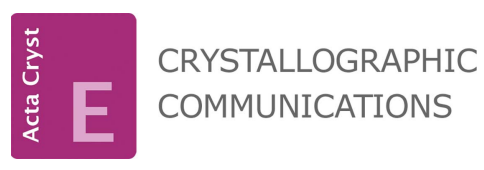

ISSN 2056-9890

Received 15 February 2019

Accepted 16 June 2019

Edited by W. T. A. Harrison, University of Aberdeen, Scotland

Keywords: Thiourea; benzamide derivative; crystal structure; Hirshfeld surface.

CCDC references: 1923234; 1923233

Supporting information: this article has supporting information at journals.iucr.org/e

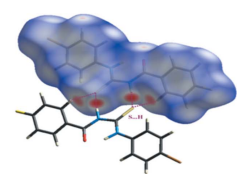

OPEN $\odot$ ACCESS

\section{Crystal structure and Hirshfeld surface analysis of $\mathrm{N}$-(2-chlorophenylcarbamothioyl)-4-fluoro- benzamide and $\mathrm{N}$-(4-bromophenylcarbamothioyl)- 4-fluorobenzamide}

Sidra Akhter, Muhammad Iqbal Choudhary, Hina Siddiqui and Sammer Yousuf*

H.E.J. Research Institute Of Chemistry, International Center for Chemical and Biological Sciences, University of Karachi, Karachi 75270, Pakistan. *Correspondence e-mail: dr.sammer.yousuf@gmail.com

The title compounds, $\mathrm{C}_{14} \mathrm{H}_{10} \mathrm{ClFN}_{2} \mathrm{OS}$ (1) and $\mathrm{C}_{14} \mathrm{H}_{10} \mathrm{BrFN}_{2} \mathrm{OS}$ (2), were synthesized by two-step reactions. The dihedral angles between the aromatic rings are 31.99 (3) and $9.17(5)^{\circ}$ for $\mathbf{1}$ and $\mathbf{2}$, respectively. Compound $\mathbf{1}$ features an intramolecular bifurcated $\mathrm{N}-\mathrm{H} \cdots(\mathrm{O}, \mathrm{Cl})$ link due to the presence of the ortho- $\mathrm{Cl}$ atom on the benzene ring, whereas 2 features an intramolecular $\mathrm{N}-$ $\mathrm{H}$. . O hydrogen bond. In the crystal of $\mathbf{1}$, inversion dimers linked by pairs of $\mathrm{N}-\mathrm{H} \cdots \mathrm{S}$ hydrogen bonds generate $R_{2}^{2}(8)$ loops. The extended structure of 2 features the same motif but an additional weak $\mathrm{C}-\mathrm{H} \cdots \mathrm{S}$ interaction links the inversion dimers into [100] double columns. Hirshfeld surface analyses indicate that the most important contributors towards the crystal packing are $\mathrm{H} \cdots \mathrm{H}$ $(26.6 \%), \mathrm{S} \cdots \mathrm{H} / \mathrm{H} . \mathrm{S}(13.8 \%)$ and $\mathrm{Cl} \cdots \mathrm{H} / \mathrm{H} \cdots \mathrm{Cl}(9.5 \%)$ contacts for $\mathbf{1}$ and $\mathrm{H} \cdots \mathrm{H}(19.7 \%), \mathrm{C} \cdots \mathrm{H} / \mathrm{H} \cdots \mathrm{C}(14.8 \%)$ and $\mathrm{Br} \cdots \mathrm{H} / \mathrm{H} \cdots \mathrm{Br}(12.4 \%)$ contacts for 2.

\section{Chemical context}

Thiourea and its derivatives show a broad range of biological activities (Solmaz et al., 2018; Saeed et al., 2018; Pandey et al., 2019). The crystal structures of many thiourea derivatives and their metal complexes have been reported (Lai et al.,2018; Contreras Aguilar et al., 2018; Fakhar et al., 2018; Mitoraj et al., 2018; Pervez et al., 2018; Hashim et al., 2017 Ghazal et al., 2019; Zhang et al., 2019). As part of our studies in this area, we now describe the syntheses, crystal structures and Hirshfeld surface analyses of the thiourea derivatives $\mathrm{N}$-(2-chlorophenylcarbamothioyl)-4-fluorobenzamide $\quad\left(\mathrm{C}_{14} \mathrm{H}_{10} \mathrm{ClFN}_{2} \mathrm{OS}, \quad\right.$ 1) and $\quad N$-(4-bromophenylcarbamothioyl)-4-fluorobenzamide $\left(\mathrm{C}_{14} \mathrm{H}_{10} \mathrm{BrFN}_{2} \mathrm{OS}, 2\right)$. The biological activities of these compounds were previously reported by Khan et al. (2018).<smiles>O=C(NC(=S)Nc1ccccc1Cl)c1ccc(F)cc1</smiles><smiles>O=C(NC(=S)Nc1ccc(Br)cc1)c1ccc(F)cc1</smiles>

\section{Structural commentary}

Compound 1 (Fig. 1) is composed of a para-fluoro-substituted $[\mathrm{C}-\mathrm{F}=1.3579(16) \AA]$ benzoyl ring linked to a ortho-chlorosubstituted phenyl ring $[\mathrm{C}-\mathrm{Cl}=1.7387(14) \AA]$ in while in 2 (Fig. 2), a para-fluoro-substituted $[\mathrm{C}-\mathrm{F}=1.350(2) \AA]$ benzoyl ring is linked to a para-bromo-substituted phenyl ring 


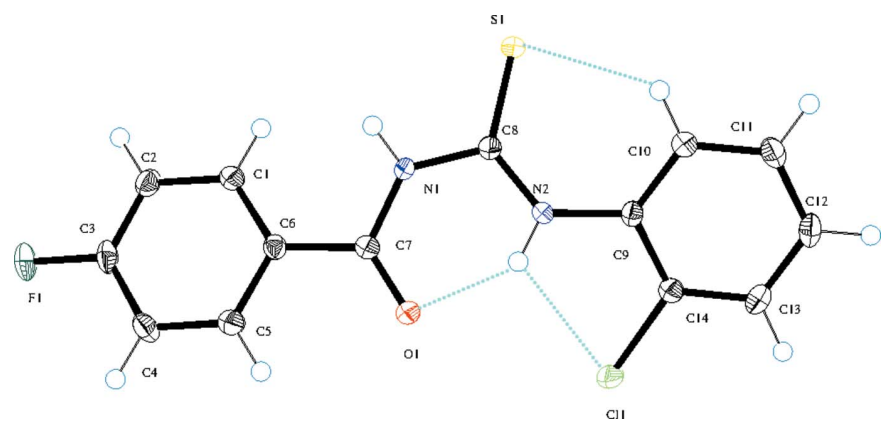

Figure 1

The molecular structure of $\mathbf{1}$ showing $50 \%$ displacement ellipsoids; the blue lines represent the intramolecular interactions.

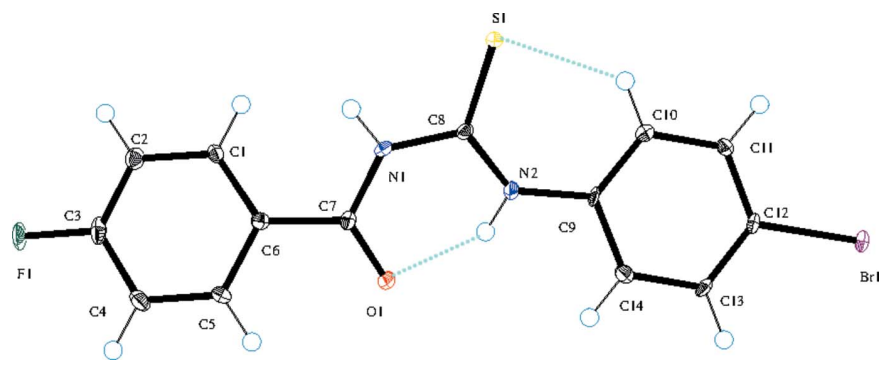

Figure 2

The molecular structure of $\mathbf{2}$ showing $50 \%$ displacement ellipsoids; the blue lines represent the intramolecular interactions.

$[\mathrm{C}-\mathrm{Br}=1.8991(17) \AA$ A $]$ via a thiourea (S1/N1/N2/C8) linkage. The benzoyl $(\mathrm{O} 1 / \mathrm{C} 1-\mathrm{C} 7)$ and phenyl rings $(\mathrm{C} 9-\mathrm{C} 14)$ are arranged about the thiourea moiety in an anti fashion having torsion angles $\mathrm{C} 8-\mathrm{N} 1-\mathrm{C} 7-\mathrm{C} 6=-170.22$ (13) and $\mathrm{C} 9-$ $\mathrm{N} 2-\mathrm{C} 8-\mathrm{S} 1=4.5(2)^{\circ}$ in compound $\mathbf{1}$, with corresponding values of $-176.01(16)$ and $3.8(3)^{\circ}$, respectively, in compound 2. The dihedral angles between the phenyl rings are 31.99 (3) and $9.17(5)^{\circ}$ in $\mathbf{1}$ and $\mathbf{2}$, respectively. Compound $\mathbf{1}$ features an intramoleclar bifurcated $\mathrm{N}-\mathrm{H} \cdots(\mathrm{O}, \mathrm{Cl})$ hydrogen bond (Table 1) due to the presence of the ortho- $\mathrm{Cl}$ atom whereas $\mathbf{2}$ has an intramolecular $\mathrm{N}-\mathrm{H} \cdots \mathrm{O}$ link (Table 2). Both structures feature an intramolecular $\mathrm{C}-\mathrm{H} \cdots \mathrm{S}$ bond, which closes an $S(6)$ ring. These intramolecular hydrogen bonds may be responsible for the anti arrangement of the aromatic rings about the thiourea linker.

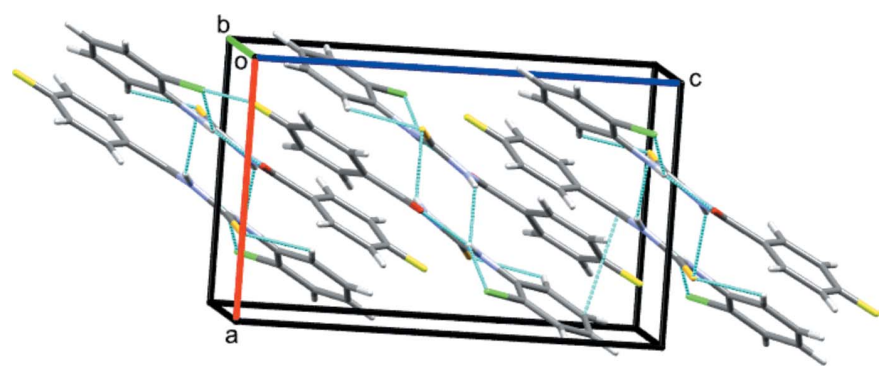

Figure 3

Partial packing diagram for 1. Light-blue lines indicate directional interactions
Table 1

Hydrogen-bond geometry $\left(\AA,^{\circ}\right)$ for $\mathbf{1}$.

\begin{tabular}{lllll}
\hline$D-\mathrm{H} \cdots A$ & $D-\mathrm{H}$ & $\mathrm{H} \cdots A$ & $D \cdots A$ & $D-\mathrm{H} \cdots A$ \\
\hline $\mathrm{C} 10-\mathrm{H} 10 \cdots \mathrm{S} 1$ & 0.95 & 2.57 & $3.1945(14)$ & 124 \\
$\mathrm{~N} 2-\mathrm{H} 1 B \cdots \mathrm{Cl}$ & $0.87(2)$ & $2.482(19)$ & $2.9246(12)$ & $112.3(14)$ \\
$\mathrm{N} 2-\mathrm{H} 1 B \cdots \mathrm{O} 1$ & $0.87(2)$ & $1.924(19)$ & $2.6600(14)$ & $141.6(17)$ \\
$\mathrm{N} 1-\mathrm{H} 1 A \cdots \mathrm{S} 1^{\mathrm{i}}$ & $0.85(2)$ & $2.67(2)$ & $3.4031(13)$ & $145.2(16)$ \\
\hline
\end{tabular}

Symmetry code: (i) $-x+1,-y+1,-z+1$.

Table 2

Hydrogen-bond geometry $\left(\AA{ }^{\circ}\right)$ for $\mathbf{2}$.

\begin{tabular}{lllll}
\hline$D-\mathrm{H} \cdots A$ & $D-\mathrm{H}$ & $\mathrm{H} \cdots A$ & $D \cdots A$ & $D-\mathrm{H} \cdots A$ \\
\hline $\mathrm{N} 1-\mathrm{H} 1 A \cdots \mathrm{S} 1^{\mathrm{i}}$ & 0.88 & 2.69 & $3.5081(15)$ & 154 \\
$\mathrm{~N} 2-\mathrm{H} 1 B \cdots \mathrm{O} 1$ & 0.88 & 1.88 & $2.610(2)$ & 139 \\
$\mathrm{C} 10-\mathrm{H} 10 \cdots \mathrm{S} 1$ & 0.95 & 2.65 & $3.2319(18)$ & 120 \\
$\mathrm{C} 1-\mathrm{H} 1 \cdots \mathrm{S} 1^{\mathrm{ii}}$ & 0.95 & 2.81 & $3.7312(18)$ & 165 \\
\hline
\end{tabular}

Symmetry codes: (i) $-x+1,-y+1,-z$; (ii) $-x,-y+1,-z$.

\section{Supramolecular features}

In the crystal of $\mathbf{1}$, inversion dimers linked by pairwise $\mathrm{N} 1-$ H1 $A \cdots \mathrm{S} 1$ hydrogen bonds (Table 1$)$ generate $R_{2}^{2}(8)$ loops (Fig. 3). The crystal of 2 features the same motif (Table 2), but an additional weak $\mathrm{C}-\mathrm{H} \cdots \mathrm{S}$ bond links the dimers into double columns propagating in the [100] direction (Fig. 4).

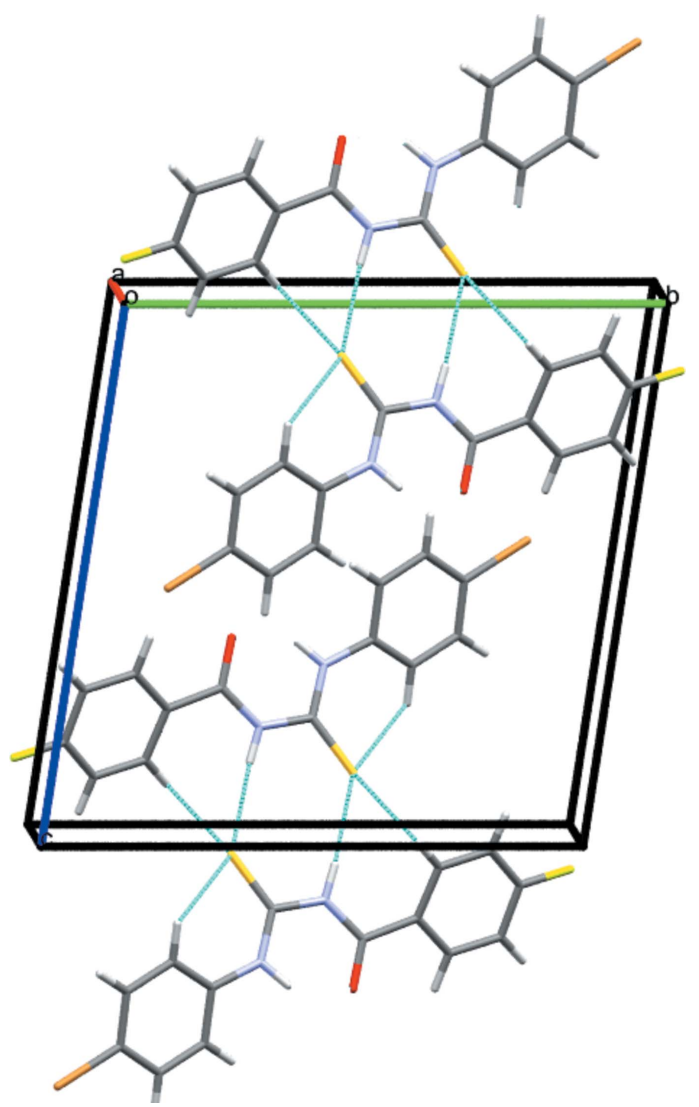

Figure 4

Partial packing diagram for 2. Light-blue lines indicate directional interactions 

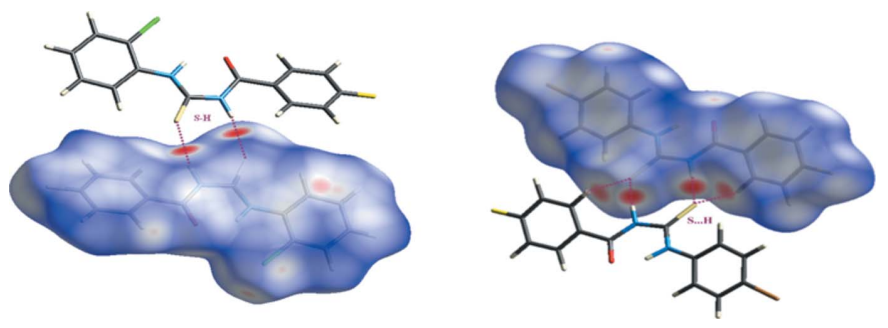

Figure 5

The Hirshfeld surfaces of $\mathbf{1}$ and $\mathbf{2}$.

\section{Database survey}

A search of Cambridge Structural Database (CSD version 5.39, update of February 2018) for compounds related to 1 and 2 yielded hits for $N$-\{[4-chloro-3-(trifluoromethyl)phenyl]carbamothioyl\}-3-methylbenzamide (CCDC deposition No. $1840069)$ and 4-chloro- $N$ - $\{[4$-chloro-3-(trifluoromethyl)phenyl]carbamothioyl benzamide (CCDC 1587395) (Zhang et al., 2019): these compounds have the same skeleton as the title compounds but with different substituents attached to the phenyl rings. In both compounds, pairwise $\mathrm{N}-\mathrm{H} \cdots \mathrm{S}$ hydrogen bonds are responsible for the formation of inversion dimers with an $R_{2}^{2}(8)$ motif, as also observed in title compounds.

\section{Hirshfeld surface analysis}

In order to further analyse the close contacts and intermolecular interactions in the crystals of $\mathbf{1}$ and 2, Hirshfeld surfaces (mapped over $d_{\text {norm }}$, curvedness and shape-index) (Fig. 5) and two-dimensional fingerprint plots (Figs. 6 and 7)

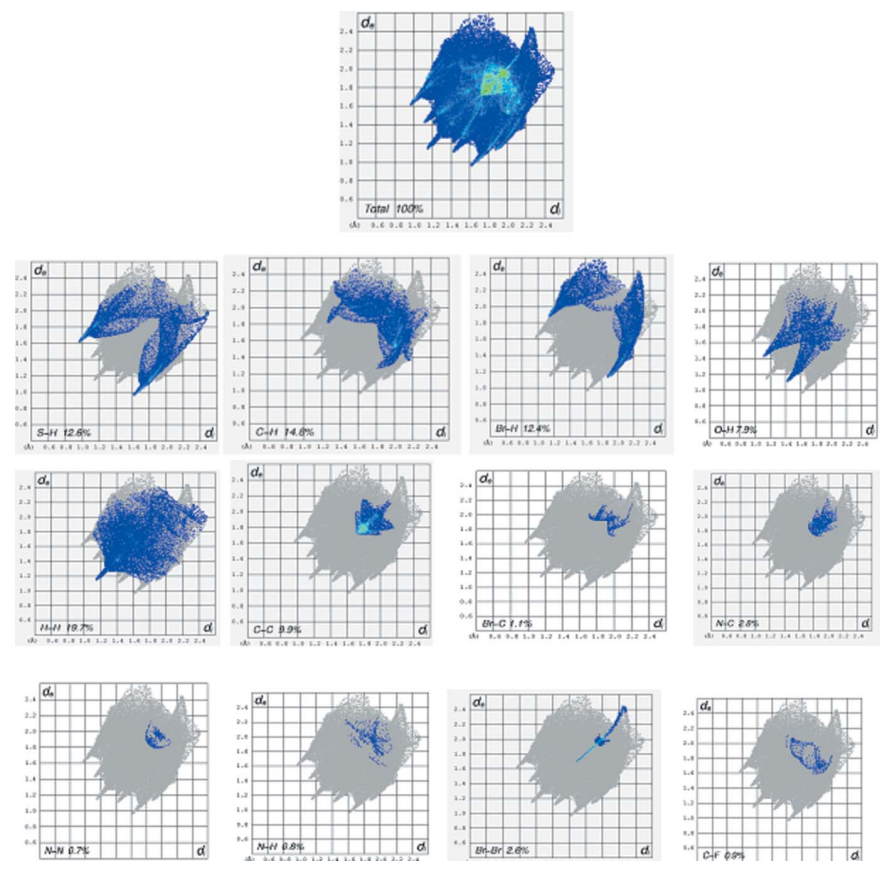

Figure 7

Two dimensional fingerprint plots for 2 .

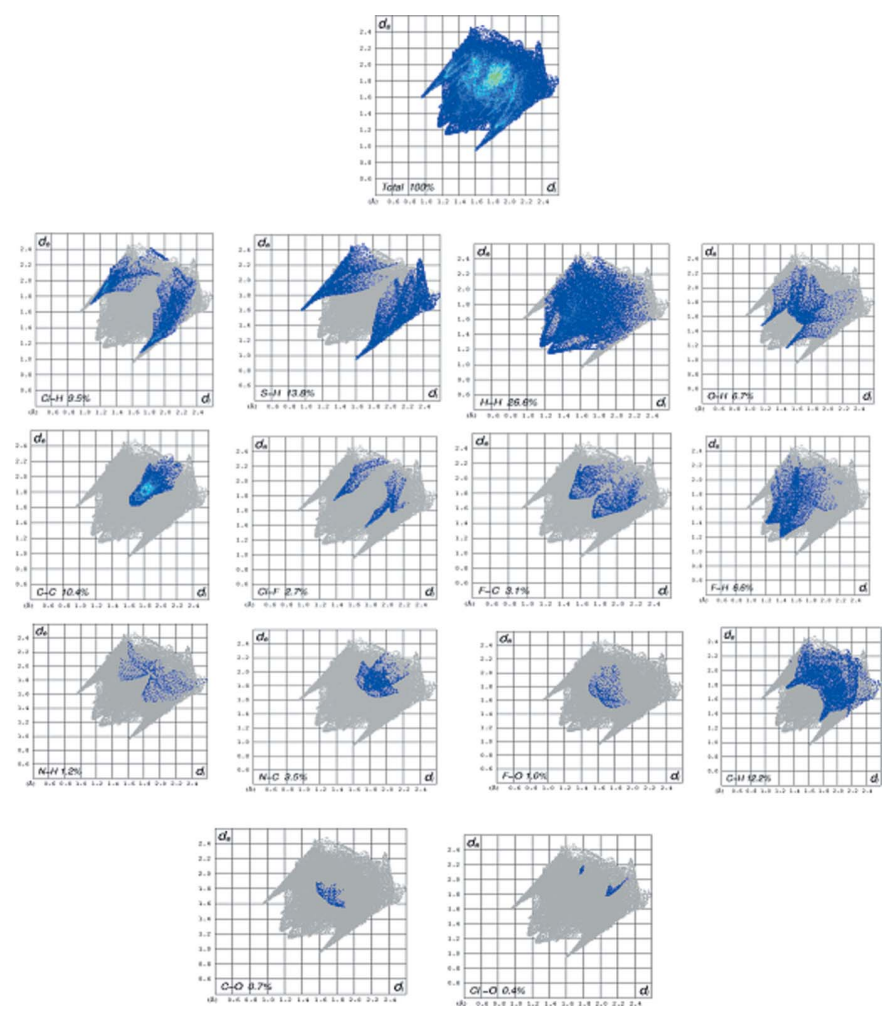

Figure 6

Two dimensional fingerprint plots for $\mathbf{1}$.

were generated using CrystalExplorer3.1 (Mackenzie et al., 2017). The fingerprint plot for $\mathbf{1}$ decomposed into individual contact types indicates that the the most significant contributions are from $\mathrm{H} \cdots \mathrm{H}$ (van der Waals) (26.6\%) contacts, followed by $\mathrm{S} \cdots \mathrm{H} / \mathrm{H} \cdots \mathrm{S}(13.8 \%), \mathrm{Cl} \cdots \mathrm{H} / \mathrm{H} \cdots \mathrm{Cl}(9.5 \%)$ $\mathrm{O} \cdots \mathrm{H} / \mathrm{H} \cdots \mathrm{O} \quad(6.7 \%), \quad \mathrm{F} \cdots \mathrm{H} / \mathrm{H} \cdots \mathrm{F} \quad(6.6 \%), \quad \mathrm{Cl} \cdots \mathrm{F} / \mathrm{F} \cdots \mathrm{Cl}$ $(3.7 \%)$ and $\mathrm{F} \cdot \mathrm{C} / \mathrm{C} \cdots \mathrm{F}(3.1 \%)$ interactions. In compound 2 , $\mathrm{H} \cdot \mathrm{H}(19.7 \%)$ (van der Waals contacts) are the most significant, followed by $\mathrm{C} \cdots \mathrm{H} / \mathrm{H} \cdots \mathrm{C} \quad(14.8 \%), \mathrm{S} \cdots \mathrm{H} / \mathrm{H} \cdots \mathrm{S}$ $(12.6 \%), \mathrm{Br} \cdots \mathrm{H} / \mathrm{H} \cdots \mathrm{Br}(12.4 \%), \mathrm{C} \cdots \mathrm{C}(9.9 \%)$ and $\mathrm{O} \cdots \mathrm{N} /$ N...O $(7.9 \%)$ interactions.

\section{Synthesis and Crystallization}

Compounds $\mathbf{1}$ and $\mathbf{2}$ were synthesized by adopting a literature procedure (Binzet et al., 2018) with slight modification: we refluxed the reactants in distilled solvents for $20 \mathrm{~min}$. instead of refluxing them in anhydrous solvents for $4 \mathrm{~h}$. In the first step, 4-fluorobenzoyle chloride $(1 \mathrm{mmol})$ and potassium thiocyanate $(1 \mathrm{mmol})$ were dissolved in acetone $(10 \mathrm{ml})$ at room temperature with constant stirring for 20 minutes to obtain a white precipitate of 4-fluorophenyl isothiocyanate. In the second step, $1 \mathrm{mmol}$ of 2-chloro phenyl aniline (for 1) or 4bromophenyl aniline (for 2) were added to the mixture and refluxed at $343 \mathrm{~K}$. Hydrochloric acid $(0.5 \mathrm{~N}, 10 \mathrm{ml})$ was added and the solution was filtered to obtain the desired products: 1 in $69 \%$ yield and $\mathbf{2}$ in $80 \%$ yield. For recrystallization, compound $\mathbf{1}$ was dissolved in a mixture of dichloromethane 
Table 3

Experimental details.

1

Crystal data

Chemical formula

$M_{\text {r }}$

Crystal system, space group

Temperature $(\mathrm{K})$

$a, b, c(\AA)$

$\alpha, \beta, \gamma\left(^{\circ}\right)$

$V\left(\AA^{3}\right)$

Z

Radiation type

$\mu\left(\mathrm{mm}^{-1}\right)$

Crystal size (mm)

Data collection

Diffractometer

Absorption correction

$T_{\min }, T_{\max }$

No. of measured, independent and

observed $[I>2 \sigma(I)]$ reflections

$R_{\text {int }}$

$(\sin \theta / \lambda)_{\max }\left(\AA^{-1}\right)$

Refinement

$R\left[F^{2}>2 \sigma\left(F^{2}\right)\right], w R\left(F^{2}\right), S$

No. of reflections

No. of parameters

$\mathrm{H}$-atom treatment

$\Delta \rho_{\max }, \Delta \rho_{\min }\left(\mathrm{e} \AA^{-3}\right)$

\section{$\mathrm{C}_{14} \mathrm{H}_{10} \mathrm{ClFN}_{2} \mathrm{OS}$}

308.75

Monoclinic, $P 2_{1} / c$

100

8.0785 (2), 12.4230 (3), 13.0772 (3)

$90,90.551(1), 90$

1312.36 (5)

4

$\mathrm{Cu} K \alpha$

4.15

$0.11 \times 0.07 \times 0.03$

Bruker APEXII CCD

Multi-scan ( $S A D A B S$; Bruker,

2000)

$0.682,0.895$

18686, 2362, 2269

0.023

0.602

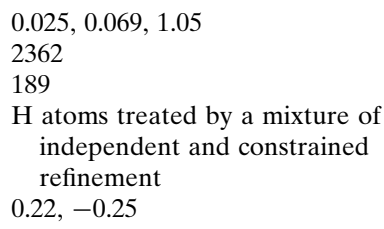

$0.025,0.069,1.05$

2362

189

$\mathrm{H}$ atoms treated by a mixture of

independent and constrained

refinement

$0.22,-0.25$

2

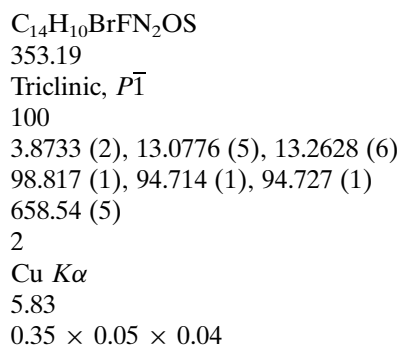

Bruker APEXII CCD

Multi-scan ( $S A D A B S$; Bruker, 2000)

$0.612,0.946$

20701, 2384, 2381

0.025

0.602

$0.025,0.110,1.10$

2384

181

$\mathrm{H}$-atom parameters constrained

$0.45,-1.46$

Computer programs: APEX2 and SAINT (Bruker, 2000), SHELXT2014 (Sheldrick, 2015a), SHELXL2016 (Sheldrick, 2015b) and SHELXTL (Sheldrick, 2008).

and methanol (1:1) while compound 2 was dissolved in dichloromethane and left for slow evaporation at room temperature to obtain colourless prisms of $\mathbf{1}$ and colourless plates of $\mathbf{2}$

\section{Data collection and Refinement}

Crystal data, data collection and structure refinement details are summarized in Table 3. The $\mathrm{C}$-bound $\mathrm{H}$ atoms atoms were positioned with idealized geometry $(\mathrm{C}-\mathrm{H}=0.93-0.97 \AA)$ and refined as riding atoms. In $\mathbf{1}$, the $\mathrm{N}$-bound $\mathrm{H}$ atoms were located in difference-Fourier maps and their positions were freely refined; in 2, the $\mathrm{N}$-bound $\mathrm{H}$ atoms were located in difference-Fourier maps and refined as riding atoms in their as-found relative positions. The constraint $U_{\text {iso }}(\mathrm{H})=$ $1.2 U_{\text {eq }}$ (carrier) was applied in all cases.

\section{Funding information}

The authors thank the Higher Education Commission of Pakistan (HEC) for financial support through research project No. 20-2830 under the National Research Program for Universities.

\section{References}

Binzet, G., Gumus, I., Dogen, A., Flörke, U., Kulcu, N. \& Arslan, H. (2018). J. Mol. Struct. 1161, 519-529.
Bruker (2000). APEX2, SAINT and SADABS. Bruker AXS Inc., Madison, Wisconsin, USA.

Contreras Aguilar, E., Echeverría, G., Piro, O., Ulic, S., Jios, J., Tuttolomondo, M. \& Pérez, H. (2018). Mol. Phys. 116, 399-413.

Fakhar, I., Hussien, N. J., Sapari, S., Bloh, A. H., Yusoff, S. F. M., Hasbullah, S. A., Yamin, B. M., Mutalib, S. A., Shihab, M. S. \& Yousif, E. (2018). J. Mol. Struct. 1159, 96-102.

Ghazal, K., Shoaib, S., Khan, M., Khan, S., Rauf, M. K., Khan, N., Badshah, A., Tahir, M. N. \& Ali, I. (2019). J. Mol. Struct. 1177, 12130.

Hashim, S. N. M., Jumal, J. \& Kassim, K. (2017). Adv. Sci. Lett. 23, 4523-4527.

Khan, M. R., Zaib, S., Rauf, M. K., Ebihara, M., Badshah, A., Zahid, M., Nadeem, M. A. \& Iqbal, J. (2018). J. Mol. Struct. 1164, 354-362.

Lai, L. C., Rahman, C. N. B. C. A., Tahir, M. I. M., Ravoof, T. B. S. A., Jotani, M. M. \& Tiekink, E. R. T. (2018). Acta Cryst. E74, 256-260.

Mackenzie, C. F., Spackman, P. R., Jayatilaka, D. \& Spackman, M. A. (2017). IUCrJ, 4, 575-587.

Mitoraj, M. P., Babashkina, M. G., Isaev, A. Y., Chichigina, Y. M., Robeyns, K., Garcia, Y. \& Safin, D. A. (2018). Cryst. Growth Des. 18, 5385-5397.

Pandey, S. K., Pratap, S., Tiwari, M. K., Marverti, G. \& Jasinski, J. P. (2019). J. Mol. Struct. 1175, 963-970.

Pervez, H., Khan, N., Iqbal, J., Zaib, S., Yaqub, M., Tahir, M. N. \& Naseer, M. M. (2018). Heterocycl. Commun. 24, 51-58.

Saeed, A., Mustafa, M. N., Zain-ul-Abideen, M., Shabir, G., Erben, M. F. \& Flörke, U. (2018). J. Sulfur Chem. pp. 1-39.

Sheldrick, G. M. (2008). Acta Cryst. A64, 112-122.

Sheldrick, G. M. (2015). Acta Cryst. C71, 3-8.

Solmaz, U., Gumus, I., Binzet, G., Celik, O., Balci, G. K., Dogen, A. \& Arslan, H. (2018). J. Coord. Chem. 71, 200-218.

Zhang, Y., Zhang, X., Qiao, L., Ding, Z., Hang, X., Qin, B., Song, J. \& Huang, J. (2019). J. Mol. Struct. 1176, 335-345. 


\section{supporting information}

Acta Cryst. (2019). E75, 1026-1029 [https://doi.org/10.1107/S2056989019008569]

Crystal structure and Hirshfeld surface analysis of $\mathrm{N}$-(2-chlorophenylcarbamothioyl)-4-fluorobenzamide and $\mathrm{N}$-(4-bromophenylcarbamothioyl)-4-fluorobenzamide

\section{Sidra Akhter, Muhammad Iqbal Choudhary, Hina Siddiqui and Sammer Yousuf}

Computing details

For both structures, data collection: APEX2 (Bruker, 2000); cell refinement: SAINT (Bruker, 2000); data reduction: SAINT (Bruker, 2000); program(s) used to solve structure: SHELXT2014 (Sheldrick, 2015a); program(s) used to refine structure: SHELXL2016 (Sheldrick, 2015b); molecular graphics: SHELXTL (Sheldrick, 2008); software used to prepare material for publication: SHELXTL (Sheldrick, 2008).

$\mathrm{N}$-(2-Chlorophenylcarbamothioyl)-4-fluorobenzamide (1)

Crystal data

$\mathrm{C}_{14} \mathrm{H}_{10} \mathrm{ClFN}_{2} \mathrm{OS}$

$M_{r}=308.75$

Monoclinic, $P 2_{1} / c$

$a=8.0785(2) \AA$

$b=12.4230(3) \AA$

$c=13.0772(3) \AA$

$\beta=90.551(1)^{\circ}$

$V=1312.36(5) \AA^{3}$

$Z=4$

$F(000)=632$

$D_{\mathrm{x}}=1.563 \mathrm{Mg} \mathrm{m}^{-3}$

$\mathrm{Cu} K \alpha$ radiation, $\lambda=1.54178 \AA$

Cell parameters from 9977 reflections

$\theta=4.9-68.3^{\circ}$

$\mu=4.15 \mathrm{~mm}^{-1}$

$T=100 \mathrm{~K}$

Prism, colourless

$0.11 \times 0.07 \times 0.03 \mathrm{~mm}$

Data collection

Bruker APEXII CCD

2362 independent reflections

diffractometer

$\omega$ scans

Absorption correction: multi-scan

(SADABS; Bruker, 2000)

$T_{\min }=0.682, T_{\max }=0.895$

18686 measured reflections

2269 reflections with $I>2 \sigma(I)$

$R_{\text {int }}=0.023$

$\theta_{\text {max }}=68.3^{\circ}, \theta_{\min }=4.9^{\circ}$

$h=-9 \rightarrow 9$

$k=-14 \rightarrow 14$

$l=-15 \rightarrow 15$

Refinement

Refinement on $F^{2}$

Least-squares matrix: full

$R\left[F^{2}>2 \sigma\left(F^{2}\right)\right]=0.025$

$w R\left(F^{2}\right)=0.069$

$S=1.05$

2362 reflections

189 parameters

0 restraints

Primary atom site location: dual

Hydrogen site location: mixed

$\mathrm{H}$ atoms treated by a mixture of independent and constrained refinement

$w=1 /\left[\sigma^{2}\left(F_{\mathrm{o}}^{2}\right)+(0.0347 P)^{2}+0.7594 P\right]$

where $P=\left(F_{\mathrm{o}}^{2}+2 F_{\mathrm{c}}^{2}\right) / 3$

$(\Delta / \sigma)_{\max }=0.001$

$\Delta \rho_{\max }=0.22 \mathrm{e}^{-3}$

$\Delta \rho_{\min }=-0.24$ e $\AA^{-3}$ 


\section{Special details}

Geometry. All esds (except the esd in the dihedral angle between two 1.s. planes) are estimated using the full covariance matrix. The cell esds are taken into account individually in the estimation of esds in distances, angles and torsion angles; correlations between esds in cell parameters are only used when they are defined by crystal symmetry. An approximate (isotropic) treatment of cell esds is used for estimating esds involving l.s. planes.

Fractional atomic coordinates and isotropic or equivalent isotropic displacement parameters $\left(\AA^{2}\right)$

\begin{tabular}{lllll}
\hline & $x$ & $y$ & $z$ & $U_{\text {iso }} * / U_{\text {eq }}$ \\
\hline S1 & $0.73325(5)$ & $0.47025(3)$ & $0.56374(3)$ & $0.02331(12)$ \\
C1 & $0.83162(5)$ & $0.03157(3)$ & $0.58416(3)$ & $0.02495(11)$ \\
F1 & $0.18907(12)$ & $0.30493(8)$ & $0.01782(7)$ & $0.0314(2)$ \\
O1 & $0.54889(12)$ & $0.15637(8)$ & $0.42244(7)$ & $0.0192(2)$ \\
N1 & $0.56777(15)$ & $0.33824(10)$ & $0.44484(9)$ & $0.0179(3)$ \\
N2 & $0.70992(14)$ & $0.25311(9)$ & $0.57516(9)$ & $0.0157(2)$ \\
C1 & $0.43467(17)$ & $0.36712(11)$ & $0.24179(10)$ & $0.0177(3)$ \\
H1 & 0.493438 & 0.425890 & 0.271153 & $0.021^{*}$ \\
C2 & $0.35330(18)$ & $0.38022(12)$ & $0.14870(11)$ & $0.0206(3)$ \\
H2 & 0.355095 & 0.447218 & 0.113699 & $0.025^{*}$ \\
C3 & $0.26979(18)$ & $0.29291(12)$ & $0.10866(10)$ & $0.0211(3)$ \\
C4 & $0.26446(18)$ & $0.19359(12)$ & $0.15557(11)$ & $0.0224(3)$ \\
H4 & 0.206152 & 0.135158 & 0.125243 & $0.027^{*}$ \\
C5 & $0.34669(18)$ & $0.18165(11)$ & $0.24822(11)$ & $0.0199(3)$ \\
H5 & 0.345811 & 0.113881 & 0.281860 & $0.024^{*}$ \\
C6 & $0.43099(16)$ & $0.26820(11)$ & $0.29281(10)$ & $0.0156(3)$ \\
C7 & $0.51917(16)$ & $0.24761(11)$ & $0.39152(10)$ & $0.0160(3)$ \\
C8 & $0.67071(16)$ & $0.34671(11)$ & $0.53083(10)$ & $0.0162(3)$ \\
C9 & $0.80473(16)$ & $0.23264(11)$ & $0.66453(10)$ & $0.0148(3)$ \\
C10 & $0.83157(17)$ & $0.30697(11)$ & $0.74300(10)$ & $0.0184(3)$ \\
H10 & 0.789298 & 0.378033 & 0.736600 & $0.022^{*}$ \\
C11 & $0.91962(17)$ & $0.27772(12)$ & $0.83031(11)$ & $0.0213(3)$ \\
H11 & 0.938625 & 0.329427 & 0.882642 & $0.026^{*}$ \\
C12 & $0.98017(18)$ & $0.17407(12)$ & $0.84215(11)$ & $0.0224(3)$ \\
H12 & 1.040289 & 0.154849 & 0.902214 & $0.027^{*}$ \\
C13 & $0.95246(18)$ & $0.09856(12)$ & $0.76576(11)$ & $0.0217(3)$ \\
H13 & 0.992398 & 0.027057 & 0.773432 & $0.026^{*}$ \\
C14 & $0.86603(17)$ & $0.12824(11)$ & $0.67813(10)$ & $0.0170(3)$ \\
H1B & $0.672(2)$ & $0.1970(16)$ & $0.5432(14)$ & $0.028(5)^{*}$ \\
H1A & $0.528(2)$ & $0.3988(16)$ & $0.4260(14)$ & $0.031(5)^{*}$ \\
& & & &
\end{tabular}

Atomic displacement parameters $\left(\AA^{2}\right)$

\begin{tabular}{lllllll}
\hline & $U^{11}$ & $U^{22}$ & $U^{33}$ & $U^{12}$ & $U^{13}$ & $U^{23}$ \\
\hline S1 & $0.0331(2)$ & $0.01172(18)$ & $0.0249(2)$ & $-0.00054(13)$ & $-0.01311(15)$ & $-0.00095(12)$ \\
C1 & $0.0406(2)$ & $0.01417(18)$ & $0.01996(19)$ & $0.00501(14)$ & $-0.00550(15)$ & $-0.00194(12)$ \\
F1 & $0.0374(5)$ & $0.0388(5)$ & $0.0178(4)$ & $0.0005(4)$ & $-0.0147(4)$ & $0.0016(4)$ \\
O1 & $0.0267(5)$ & $0.0138(5)$ & $0.0170(5)$ & $-0.0006(4)$ & $-0.0048(4)$ & $0.0011(4)$ \\
N1 & $0.0248(6)$ & $0.0126(6)$ & $0.0161(6)$ & $0.0039(5)$ & $-0.0079(5)$ & $-0.0007(4)$
\end{tabular}




$\begin{array}{lllllll}\mathrm{N} 2 & 0.0206(6) & 0.0125(6) & 0.0141(6) & 0.0000(5) & -0.0046(4) & -0.0015(4) \\ \text { C1 } & 0.0208(7) & 0.0165(6) & 0.0158(7) & 0.0002(5) & -0.0008(5) & -0.0019(5) \\ \text { C2 } & 0.0257(7) & 0.0196(7) & 0.0166(7) & 0.0034(6) & -0.0016(6) & 0.0024(5) \\ \text { C3 } & 0.0209(7) & 0.0304(8) & 0.0119(7) & 0.0041(6) & -0.0042(5) & -0.0015(6) \\ \text { C4 } & 0.0241(7) & 0.0243(8) & 0.0188(7) & -0.0040(6) & -0.0045(6) & -0.0048(6) \\ \text { C5 } & 0.0240(7) & 0.0170(7) & 0.0187(7) & -0.0012(6) & -0.0017(5) & -0.0007(5) \\ \text { C6 } & 0.0158(6) & 0.0171(7) & 0.0137(6) & 0.0017(5) & -0.0004(5) & -0.0012(5) \\ \text { C7 } & 0.0171(6) & 0.0152(7) & 0.0158(7) & 0.0000(5) & -0.0002(5) & -0.0011(5) \\ \text { C8 } & 0.0184(6) & 0.0150(6) & 0.0152(6) & 0.0020(5) & -0.0023(5) & -0.0010(5) \\ \text { C9 } & 0.0139(6) & 0.0169(6) & 0.0135(6) & -0.0009(5) & -0.0008(5) & 0.0022(5) \\ \text { C10 } & 0.0208(7) & 0.0169(7) & 0.0175(7) & -0.0002(5) & -0.0020(5) & -0.0003(5) \\ \text { C11 } & 0.0221(7) & 0.0250(7) & 0.0167(7) & -0.0030(6) & -0.0034(5) & -0.0019(6) \\ \text { C12 } & 0.0211(7) & 0.0292(8) & 0.0169(7) & 0.0001(6) & -0.0055(5) & 0.0048(6) \\ \text { C13 } & 0.0226(7) & 0.0211(7) & 0.0215(7) & 0.0039(6) & -0.0024(6) & 0.0053(6) \\ \text { C14 } & 0.0189(6) & 0.0160(7) & 0.0161(6) & 0.0000(5) & -0.0001(5) & -0.0007(5)\end{array}$

Geometric parameters $\left(A,{ }^{o}\right)$

\begin{tabular}{|c|c|c|c|}
\hline $\mathrm{S} 1-\mathrm{C} 8$ & $1.6709(14)$ & $\mathrm{C} 4-\mathrm{C} 5$ & $1.384(2)$ \\
\hline $\mathrm{Cl}-\mathrm{C} 14$ & $1.7387(14)$ & $\mathrm{C} 4-\mathrm{H} 4$ & 0.9500 \\
\hline $\mathrm{F} 1-\mathrm{C} 3$ & $1.3579(16)$ & $\mathrm{C} 5-\mathrm{C} 6$ & $1.3972(19)$ \\
\hline $\mathrm{O} 1-\mathrm{C} 7$ & $1.2264(17)$ & $\mathrm{C} 5-\mathrm{H} 5$ & 0.9500 \\
\hline $\mathrm{N} 1-\mathrm{C} 7$ & $1.3794(18)$ & $\mathrm{C} 6-\mathrm{C} 7$ & $1.4904(18)$ \\
\hline $\mathrm{N} 1-\mathrm{C} 8$ & $1.3961(17)$ & $\mathrm{C} 9-\mathrm{C} 10$ & $1.3959(19)$ \\
\hline $\mathrm{N} 1-\mathrm{H} 1 \mathrm{~A}$ & $0.85(2)$ & $\mathrm{C} 9-\mathrm{C} 14$ & $1.3990(19)$ \\
\hline $\mathrm{N} 2-\mathrm{C} 8$ & $1.3360(18)$ & $\mathrm{C} 10-\mathrm{C} 11$ & $1.3879(19)$ \\
\hline $\mathrm{N} 2-\mathrm{C} 9$ & $1.4141(17)$ & $\mathrm{C} 10-\mathrm{H} 10$ & 0.9500 \\
\hline $\mathrm{N} 2-\mathrm{H} 1 \mathrm{~B}$ & $0.87(2)$ & $\mathrm{C} 11-\mathrm{C} 12$ & $1.385(2)$ \\
\hline $\mathrm{C} 1-\mathrm{C} 2$ & $1.3875(19)$ & $\mathrm{C} 11-\mathrm{H} 11$ & 0.9500 \\
\hline $\mathrm{C} 1-\mathrm{C} 6$ & $1.3987(19)$ & $\mathrm{C} 12-\mathrm{C} 13$ & $1.387(2)$ \\
\hline $\mathrm{C} 1-\mathrm{H} 1$ & 0.9500 & $\mathrm{C} 12-\mathrm{H} 12$ & 0.9500 \\
\hline $\mathrm{C} 2-\mathrm{C} 3$ & $1.378(2)$ & $\mathrm{C} 13-\mathrm{C} 14$ & $1.3860(19)$ \\
\hline $\mathrm{C} 2-\mathrm{H} 2$ & 0.9500 & $\mathrm{C} 13-\mathrm{H} 13$ & 0.9500 \\
\hline $\mathrm{C} 3-\mathrm{C} 4$ & $1.379(2)$ & & \\
\hline $\mathrm{C} 7-\mathrm{N} 1-\mathrm{C} 8$ & $129.28(12)$ & $\mathrm{O} 1-\mathrm{C} 7-\mathrm{N} 1$ & $122.26(12)$ \\
\hline $\mathrm{C} 7-\mathrm{N} 1-\mathrm{H} 1 \mathrm{~A}$ & $117.8(13)$ & $\mathrm{O} 1-\mathrm{C} 7-\mathrm{C} 6$ & $122.32(12)$ \\
\hline $\mathrm{C} 8-\mathrm{N} 1-\mathrm{H} 1 \mathrm{~A}$ & $112.8(13)$ & $\mathrm{N} 1-\mathrm{C} 7-\mathrm{C} 6$ & $115.41(12)$ \\
\hline $\mathrm{C} 8-\mathrm{N} 2-\mathrm{C} 9$ & $129.75(12)$ & $\mathrm{N} 2-\mathrm{C} 8-\mathrm{N} 1$ & $114.89(12)$ \\
\hline $\mathrm{C} 8-\mathrm{N} 2-\mathrm{H} 1 \mathrm{~B}$ & $114.1(12)$ & $\mathrm{N} 2-\mathrm{C} 8-\mathrm{S} 1$ & $128.15(10)$ \\
\hline $\mathrm{C} 9-\mathrm{N} 2-\mathrm{H} 1 \mathrm{~B}$ & $116.1(12)$ & $\mathrm{N} 1-\mathrm{C} 8-\mathrm{S} 1$ & $116.94(10)$ \\
\hline $\mathrm{C} 2-\mathrm{C} 1-\mathrm{C} 6$ & $120.64(13)$ & $\mathrm{C} 10-\mathrm{C} 9-\mathrm{C} 14$ & $117.87(12)$ \\
\hline $\mathrm{C} 2-\mathrm{C} 1-\mathrm{H} 1$ & 119.7 & $\mathrm{C} 10-\mathrm{C} 9-\mathrm{N} 2$ & $124.58(12)$ \\
\hline $\mathrm{C} 6-\mathrm{C} 1-\mathrm{H} 1$ & 119.7 & $\mathrm{C} 14-\mathrm{C} 9-\mathrm{N} 2$ & $117.39(12)$ \\
\hline $\mathrm{C} 3-\mathrm{C} 2-\mathrm{C} 1$ & $117.84(13)$ & $\mathrm{C} 11-\mathrm{C} 10-\mathrm{C} 9$ & $120.42(13)$ \\
\hline $\mathrm{C} 3-\mathrm{C} 2-\mathrm{H} 2$ & 121.1 & $\mathrm{C} 11-\mathrm{C} 10-\mathrm{H} 10$ & 119.8 \\
\hline $\mathrm{C} 1-\mathrm{C} 2-\mathrm{H} 2$ & 121.1 & $\mathrm{C} 9-\mathrm{C} 10-\mathrm{H} 10$ & 119.8 \\
\hline $\mathrm{F} 1-\mathrm{C} 3-\mathrm{C} 2$ & $118.37(13)$ & $\mathrm{C} 12-\mathrm{C} 11-\mathrm{C} 10$ & $120.86(13)$ \\
\hline
\end{tabular}




\begin{tabular}{|c|c|c|c|}
\hline $\mathrm{F} 1-\mathrm{C} 3-\mathrm{C} 4$ & $118.09(13)$ & $\mathrm{C} 12-\mathrm{C} 11-\mathrm{H} 11$ & 119.6 \\
\hline $\mathrm{C} 2-\mathrm{C} 3-\mathrm{C} 4$ & $123.54(13)$ & $\mathrm{C} 10-\mathrm{C} 11-\mathrm{H} 11$ & 119.6 \\
\hline $\mathrm{C} 3-\mathrm{C} 4-\mathrm{C} 5$ & $117.94(13)$ & $\mathrm{C} 11-\mathrm{C} 12-\mathrm{C} 13$ & $119.58(13)$ \\
\hline $\mathrm{C} 3-\mathrm{C} 4-\mathrm{H} 4$ & 121.0 & $\mathrm{C} 11-\mathrm{C} 12-\mathrm{H} 12$ & 120.2 \\
\hline $\mathrm{C} 5-\mathrm{C} 4-\mathrm{H} 4$ & 121.0 & $\mathrm{C} 13-\mathrm{C} 12-\mathrm{H} 12$ & 120.2 \\
\hline $\mathrm{C} 4-\mathrm{C} 5-\mathrm{C} 6$ & $120.72(13)$ & $\mathrm{C} 14-\mathrm{C} 13-\mathrm{C} 12$ & $119.47(13)$ \\
\hline $\mathrm{C} 4-\mathrm{C} 5-\mathrm{H} 5$ & 119.6 & $\mathrm{C} 14-\mathrm{C} 13-\mathrm{H} 13$ & 120.3 \\
\hline $\mathrm{C} 6-\mathrm{C} 5-\mathrm{H} 5$ & 119.6 & $\mathrm{C} 12-\mathrm{C} 13-\mathrm{H} 13$ & 120.3 \\
\hline $\mathrm{C} 5-\mathrm{C} 6-\mathrm{C} 1$ & $119.30(12)$ & $\mathrm{C} 13-\mathrm{C} 14-\mathrm{C} 9$ & $121.78(13)$ \\
\hline $\mathrm{C} 5-\mathrm{C} 6-\mathrm{C} 7$ & $117.15(12)$ & $\mathrm{C} 13-\mathrm{C} 14-\mathrm{Cl}$ & $118.45(11)$ \\
\hline $\mathrm{C} 1-\mathrm{C} 6-\mathrm{C} 7$ & $123.49(12)$ & $\mathrm{C} 9-\mathrm{C} 14-\mathrm{Cl}$ & $119.76(10)$ \\
\hline $\mathrm{C} 6-\mathrm{C} 1-\mathrm{C} 2-\mathrm{C} 3$ & $-0.1(2)$ & $\mathrm{C} 9-\mathrm{N} 2-\mathrm{C} 8-\mathrm{S} 1$ & $4.5(2)$ \\
\hline $\mathrm{C} 1-\mathrm{C} 2-\mathrm{C} 3-\mathrm{F} 1$ & $179.70(12)$ & $\mathrm{C} 7-\mathrm{N} 1-\mathrm{C} 8-\mathrm{N} 2$ & $-9.6(2)$ \\
\hline $\mathrm{C} 1-\mathrm{C} 2-\mathrm{C} 3-\mathrm{C} 4$ & $-0.7(2)$ & $\mathrm{C} 7-\mathrm{N} 1-\mathrm{C} 8-\mathrm{S} 1$ & $168.84(11)$ \\
\hline $\mathrm{F} 1-\mathrm{C} 3-\mathrm{C} 4-\mathrm{C} 5$ & $-179.92(12)$ & $\mathrm{C} 8-\mathrm{N} 2-\mathrm{C} 9-\mathrm{C} 10$ & $22.4(2)$ \\
\hline $\mathrm{C} 2-\mathrm{C} 3-\mathrm{C} 4-\mathrm{C} 5$ & $0.5(2)$ & $\mathrm{C} 8-\mathrm{N} 2-\mathrm{C} 9-\mathrm{C} 14$ & $-162.20(13)$ \\
\hline $\mathrm{C} 3-\mathrm{C} 4-\mathrm{C} 5-\mathrm{C} 6$ & $0.6(2)$ & $\mathrm{C} 14-\mathrm{C} 9-\mathrm{C} 10-\mathrm{C} 11$ & $1.4(2)$ \\
\hline $\mathrm{C} 4-\mathrm{C} 5-\mathrm{C} 6-\mathrm{C} 1$ & $-1.3(2)$ & $\mathrm{N} 2-\mathrm{C} 9-\mathrm{C} 10-\mathrm{C} 11$ & $176.73(13)$ \\
\hline $\mathrm{C} 4-\mathrm{C} 5-\mathrm{C} 6-\mathrm{C} 7$ & $-178.72(13)$ & $\mathrm{C} 9-\mathrm{C} 10-\mathrm{C} 11-\mathrm{C} 12$ & $-1.1(2)$ \\
\hline $\mathrm{C} 2-\mathrm{C} 1-\mathrm{C} 6-\mathrm{C} 5$ & $1.1(2)$ & $\mathrm{C} 10-\mathrm{C} 11-\mathrm{C} 12-\mathrm{C} 13$ & $0.0(2)$ \\
\hline $\mathrm{C} 2-\mathrm{C} 1-\mathrm{C} 6-\mathrm{C} 7$ & $178.32(13)$ & $\mathrm{C} 11-\mathrm{C} 12-\mathrm{C} 13-\mathrm{C} 14$ & $0.7(2)$ \\
\hline $\mathrm{C} 8-\mathrm{N} 1-\mathrm{C} 7-\mathrm{O} 1$ & $8.6(2)$ & $\mathrm{C} 12-\mathrm{C} 13-\mathrm{C} 14-\mathrm{C} 9$ & $-0.3(2)$ \\
\hline $\mathrm{C} 8-\mathrm{N} 1-\mathrm{C} 7-\mathrm{C} 6$ & $-170.22(13)$ & $\mathrm{C} 12-\mathrm{C} 13-\mathrm{C} 14-\mathrm{Cl}$ & $-179.45(11)$ \\
\hline $\mathrm{C} 5-\mathrm{C} 6-\mathrm{C} 7-\mathrm{O} 1$ & $15.54(19)$ & $\mathrm{C} 10-\mathrm{C} 9-\mathrm{C} 14-\mathrm{C} 13$ & $-0.7(2)$ \\
\hline $\mathrm{C} 1-\mathrm{C} 6-\mathrm{C} 7-\mathrm{O} 1$ & $-161.73(13)$ & $\mathrm{N} 2-\mathrm{C} 9-\mathrm{C} 14-\mathrm{C} 13$ & $-176.39(12)$ \\
\hline $\mathrm{C} 5-\mathrm{C} 6-\mathrm{C} 7-\mathrm{N} 1$ & $-165.60(12)$ & $\mathrm{C} 10-\mathrm{C} 9-\mathrm{C} 14-\mathrm{Cl}$ & $178.42(10)$ \\
\hline $\mathrm{C} 1-\mathrm{C} 6-\mathrm{C} 7-\mathrm{N} 1$ & $17.14(19)$ & $\mathrm{N} 2-\mathrm{C} 9-\mathrm{C} 14-\mathrm{Cl}$ & $2.72(17)$ \\
\hline $\mathrm{C} 9-\mathrm{N} 2-\mathrm{C} 8-\mathrm{N} 1$ & $-177.23(12)$ & & \\
\hline
\end{tabular}

Hydrogen-bond geometry $\left(\AA,{ }^{\circ}\right)$

\begin{tabular}{lllll}
\hline$D-\mathrm{H} \cdots A$ & $D-\mathrm{H}$ & $\mathrm{H} \cdots A$ & $D \cdots A$ & $D-\mathrm{H} \cdots A$ \\
\hline $\mathrm{C} 10-\mathrm{H} 10 \cdots \mathrm{S} 1$ & 0.95 & 2.57 & $3.1945(14)$ & 124 \\
$\mathrm{~N} 2-\mathrm{H} 1 B^{\cdots} \mathrm{Cl}$ & $0.87(2)$ & $2.482(19)$ & $2.9246(12)$ & $112.3(14)$ \\
$\mathrm{N} 2-\mathrm{H} 1 B \cdots \mathrm{O} 1$ & $0.87(2)$ & $1.924(19)$ & $2.6600(14)$ & $141.6(17)$ \\
$\mathrm{N} 1-\mathrm{H} 1 A^{\cdots} \mathrm{S} 1^{\mathrm{i}}$ & $0.85(2)$ & $2.67(2)$ & $3.4031(13)$ & $145.2(16)$ \\
\hline
\end{tabular}

Symmetry code: (i) $-x+1,-y+1,-z+1$.

$\mathrm{N}$-(4-Bromophenylcarbamothioyl)-4-fluorobenzamide (2)

Crystal data

$\mathrm{C}_{14} \mathrm{H}_{10} \mathrm{BrFN}_{2} \mathrm{OS}$

$M_{r}=353.19$

Triclinic, $P \overline{1}$

$a=3.8733(2) \AA$

$b=13.0776(5) \AA$

$c=13.2628(6) \AA$

$\alpha=98.817(1)^{\circ}$

$$
\begin{aligned}
& \beta=94.714(1)^{\circ} \\
& \gamma=94.727(1)^{\circ} \\
& V=658.54(5) \AA^{3} \\
& Z=2 \\
& F(000)=348 \\
& D_{\mathrm{x}}=1.771 \mathrm{Mg} \mathrm{m}^{-3} \\
& \mathrm{Cu} K \alpha \text { radiation, } \lambda=1.54178 \AA
\end{aligned}
$$


Cell parameters from 9945 reflections

$\theta=3.4-68.2^{\circ}$

$\mu=5.83 \mathrm{~mm}^{-1}$

Data collection

Bruker APEXII CCD

diffractometer

$\varphi$ and $\omega$ scans

Absorption correction: multi-scan

(SADABS; Bruker, 2000)

$T_{\min }=0.612, T_{\max }=0.946$

20701 measured reflections

\section{Refinement}

Refinement on $F^{2}$

Least-squares matrix: full

$R\left[F^{2}>2 \sigma\left(F^{2}\right)\right]=0.025$

$w R\left(F^{2}\right)=0.110$

$S=1.10$

2384 reflections

181 parameters

0 restraints

Primary atom site location: dual
$T=100 \mathrm{~K}$

Plate, colourless

$0.35 \times 0.05 \times 0.04 \mathrm{~mm}$

2384 independent reflections

2381 reflections with $I>2 \sigma(I)$

$R_{\text {int }}=0.025$

$\theta_{\max }=68.2^{\circ}, \theta_{\min }=3.4^{\circ}$

$h=-4 \rightarrow 4$

$k=-15 \rightarrow 15$

$l=-15 \rightarrow 15$

Hydrogen site location: inferred from neighbouring sites

$\mathrm{H}$-atom parameters constrained

$w=1 /\left[\sigma^{2}\left(F_{\mathrm{o}}^{2}\right)+(0.1 P)^{2}\right]$

where $P=\left(F_{\mathrm{o}}{ }^{2}+2 F_{\mathrm{c}}{ }^{2}\right) / 3$

$(\Delta / \sigma)_{\max }=0.001$

$\Delta \rho_{\max }=0.45$ e $\AA^{-3}$

$\Delta \rho_{\min }=-1.46 \mathrm{e} \AA^{-3}$

Special details

Geometry. All esds (except the esd in the dihedral angle between two 1.s. planes) are estimated using the full covariance matrix. The cell esds are taken into account individually in the estimation of esds in distances, angles and torsion angles; correlations between esds in cell parameters are only used when they are defined by crystal symmetry. An approximate (isotropic) treatment of cell esds is used for estimating esds involving 1.s. planes.

Fractional atomic coordinates and isotropic or equivalent isotropic displacement parameters $\left(\AA^{2}\right)$

\begin{tabular}{lllll}
\hline & $x$ & $y$ & $z$ & $U_{\text {iso }} * U_{\text {eq }}$ \\
\hline Br1 & $-0.39350(4)$ & $0.11758(2)$ & $0.46689(2)$ & $0.01272(16)$ \\
O1 & $0.4560(4)$ & $0.66092(11)$ & $0.32207(10)$ & $0.0175(3)$ \\
N1 & $0.3835(4)$ & $0.58122(12)$ & $0.15534(11)$ & $0.0106(3)$ \\
H1A & 0.419261 & 0.590591 & 0.092584 & $0.013^{*}$ \\
N2 & $0.1544(4)$ & $0.47331(12)$ & $0.26027(11)$ & $0.0111(3)$ \\
H1B & 0.187274 & 0.531645 & 0.304308 & $0.013^{*}$ \\
F1 & $0.9679(3)$ & $1.04344(9)$ & $0.12214(9)$ & $0.0205(3)$ \\
S1 & $0.18617(10)$ & $0.38973(3)$ & $0.06266(3)$ & $0.00989(18)$ \\
C1 & $0.5195(4)$ & $0.78724(14)$ & $0.09959(14)$ & $0.0092(4)$ \\
H1 & 0.376290 & 0.737919 & 0.050359 & $0.011^{*}$ \\
C2 & $0.6398(5)$ & $0.88103(14)$ & $0.07394(14)$ & $0.0129(4)$ \\
H2 & 0.581012 & 0.896819 & 0.007460 & $0.016^{*}$ \\
C3 & $0.8466(5)$ & $0.95143(15)$ & $0.14634(15)$ & $0.0146(4)$ \\
C4 & $0.9345(5)$ & $0.93323(14)$ & $0.24544(14)$ & $0.0137(4)$ \\
H4 & 1.074144 & 0.983856 & 0.294279 & $0.016^{*}$ \\
C5 & $0.8117(5)$ & $0.83897(14)$ & $0.27036(14)$ & $0.0116(4)$ \\
H5 & 0.866416 & 0.824564 & 0.337592 & $0.014^{*}$ \\
C6 & $0.6063(5)$ & $0.76392(14)$ & $0.19744(13)$ & $0.0101(4)$
\end{tabular}




$\begin{array}{lllll}\text { C7 } & 0.4782(5) & 0.66558(14) & 0.23084(14) & 0.0105(4) \\ \text { C9 } & 0.0233(4) & 0.38653(13) & 0.30136(13) & 0.0086(4) \\ \text { C8 } & 0.2378(4) & 0.48253(14) & 0.16562(13) & 0.0085(4) \\ \text { C10 } & -0.1837(5) & 0.30056(14) & 0.24536(13) & 0.0115(4) \\ \text { H10 } & -0.240925 & 0.296827 & 0.173769 & 0.014^{*} \\ \text { C11 } & -0.3051(4) & 0.22064(14) & 0.29513(13) & 0.0105(4) \\ \text { H11 } & -0.444455 & 0.161739 & 0.257544 & 0.013^{*} \\ \text { C12 } & -0.2224(5) & 0.22707(13) & 0.39998(14) & 0.0102(4) \\ \text { C13 } & -0.0217(5) & 0.31221(13) & 0.45694(13) & 0.0109(4) \\ \text { H13 } & 0.031180 & 0.315998 & 0.528723 & 0.013^{*} \\ \text { C14 } & 0.0999(5) & 0.39136(15) & 0.40755(14) & 0.0114(4) \\ \text { H14 } & 0.237633 & 0.450144 & 0.445916 & 0.014^{*}\end{array}$

Atomic displacement parameters $\left(\AA^{2}\right)$

\begin{tabular}{lllllll}
\hline & $U^{11}$ & $U^{22}$ & $U^{33}$ & $U^{12}$ & $U^{13}$ & $U^{23}$ \\
\hline Br1 & $0.0169(2)$ & $0.0096(2)$ & $0.0125(2)$ & $-0.00227(13)$ & $0.00215(13)$ & $0.00588(13)$ \\
O1 & $0.0312(8)$ & $0.0122(7)$ & $0.0076(7)$ & $-0.0065(6)$ & $0.0033(6)$ & $0.0011(5)$ \\
N1 & $0.0154(8)$ & $0.0108(8)$ & $0.0057(7)$ & $0.0005(6)$ & $0.0007(6)$ & $0.0022(6)$ \\
N2 & $0.0170(8)$ & $0.0073(7)$ & $0.0090(7)$ & $-0.0001(6)$ & $0.0021(6)$ & $0.0019(6)$ \\
F1 & $0.0328(7)$ & $0.0078(6)$ & $0.0213(7)$ & $-0.0054(5)$ & $0.0078(5)$ & $0.0051(5)$ \\
S1 & $0.0140(3)$ & $0.0082(3)$ & $0.0069(3)$ & $-0.00094(19)$ & $0.00084(19)$ & $0.00050(19)$ \\
C1 & $0.0092(8)$ & $0.0080(9)$ & $0.0102(8)$ & $-0.0004(6)$ & $0.0023(6)$ & $0.0005(7)$ \\
C2 & $0.0161(9)$ & $0.0103(9)$ & $0.0133(9)$ & $0.0022(7)$ & $0.0031(7)$ & $0.0031(7)$ \\
C3 & $0.0197(9)$ & $0.0068(9)$ & $0.0194(10)$ & $0.0022(7)$ & $0.0119(8)$ & $0.0033(7)$ \\
C4 & $0.0149(9)$ & $0.0097(9)$ & $0.0148(9)$ & $-0.0026(7)$ & $0.0020(7)$ & $-0.0021(7)$ \\
C5 & $0.0144(8)$ & $0.0095(9)$ & $0.0093(8)$ & $-0.0016(7)$ & $-0.0006(7)$ & $-0.0012(7)$ \\
C6 & $0.0106(8)$ & $0.0103(9)$ & $0.0089(9)$ & $-0.0002(7)$ & $0.0008(6)$ & $0.0008(7)$ \\
C7 & $0.0118(8)$ & $0.0104(9)$ & $0.0098(9)$ & $-0.0013(7)$ & $0.0013(6)$ & $0.0037(7)$ \\
C9 & $0.0120(8)$ & $0.0070(9)$ & $0.0086(8)$ & $0.0005(7)$ & $0.0048(6)$ & $0.0047(6)$ \\
C8 & $0.0103(8)$ & $0.0086(8)$ & $0.0071(8)$ & $0.0020(7)$ & $-0.0002(6)$ & $0.0026(6)$ \\
C10 & $0.0137(8)$ & $0.0120(9)$ & $0.0088(8)$ & $0.0001(7)$ & $-0.0005(7)$ & $0.0028(6)$ \\
C11 & $0.0116(8)$ & $0.0092(9)$ & $0.0097(8)$ & $-0.0028(6)$ & $0.0007(6)$ & $0.0004(6)$ \\
C12 & $0.0128(8)$ & $0.0080(9)$ & $0.0112(8)$ & $0.0005(6)$ & $0.0023(7)$ & $0.0056(6)$ \\
C13 & $0.0138(8)$ & $0.0132(9)$ & $0.0067(8)$ & $-0.0003(7)$ & $0.0018(7)$ & $0.0048(6)$ \\
C14 & $0.0130(8)$ & $0.0092(9)$ & $0.0112(9)$ & $-0.0007(6)$ & $0.0011(7)$ & $0.0002(6)$ \\
& & & & & & \\
\hline
\end{tabular}

Geometric parameters $\left(\AA,{ }^{\circ}\right)$

\begin{tabular}{llll}
\hline $\mathrm{Br} 1-\mathrm{C} 12$ & $1.8991(17)$ & $\mathrm{C} 4-\mathrm{C} 5$ & $1.382(3)$ \\
$\mathrm{O} 1-\mathrm{C} 7$ & $1.230(2)$ & $\mathrm{C} 4-\mathrm{H} 4$ & 0.9500 \\
$\mathrm{~N} 1-\mathrm{C} 7$ & $1.374(2)$ & $\mathrm{C} 5-\mathrm{C} 6$ & $1.409(2)$ \\
$\mathrm{N} 1-\mathrm{C} 8$ & $1.396(2)$ & $\mathrm{C} 5-\mathrm{H} 5$ & 0.9500 \\
$\mathrm{~N} 1-\mathrm{H} 1 \mathrm{~A}$ & 0.8800 & $\mathrm{C} 6-\mathrm{C} 7$ & $1.484(3)$ \\
$\mathrm{N} 2-\mathrm{C} 8$ & $1.342(2)$ & $\mathrm{C} 9-\mathrm{C} 10$ & $1.399(2)$ \\
$\mathrm{N} 2-\mathrm{C} 9$ & $1.408(2)$ & $\mathrm{C} 9-\mathrm{C} 14$ & $1.406(3)$ \\
$\mathrm{N} 2-\mathrm{H} 1 \mathrm{~B}$ & 0.8800 & $\mathrm{C} 10-\mathrm{C} 11$ & $1.390(3)$ \\
$\mathrm{F} 1-\mathrm{C} 3$ & $1.350(2)$ & $\mathrm{C} 10-\mathrm{H} 10$ & 0.9500
\end{tabular}




\begin{tabular}{|c|c|c|c|}
\hline $\mathrm{S} 1-\mathrm{C} 8$ & $1.6687(18)$ & $\mathrm{C} 11-\mathrm{C} 12$ & $1.389(2)$ \\
\hline $\mathrm{C} 1-\mathrm{C} 2$ & $1.377(3)$ & $\mathrm{C} 11-\mathrm{H} 11$ & 0.9500 \\
\hline $\mathrm{C} 1-\mathrm{C} 6$ & $1.399(3)$ & $\mathrm{C} 12-\mathrm{C} 13$ & $1.385(3)$ \\
\hline $\mathrm{C} 1-\mathrm{H} 1$ & 0.9500 & $\mathrm{C} 13-\mathrm{C} 14$ & $1.379(3)$ \\
\hline $\mathrm{C} 2-\mathrm{C} 3$ & $1.375(3)$ & $\mathrm{C} 13-\mathrm{H} 13$ & 0.9500 \\
\hline $\mathrm{C} 2-\mathrm{H} 2$ & 0.9500 & C14-H14 & 0.9500 \\
\hline $\mathrm{C} 3-\mathrm{C} 4$ & $1.391(3)$ & & \\
\hline $\mathrm{C} 7-\mathrm{N} 1-\mathrm{C} 8$ & $128.20(16)$ & $\mathrm{O} 1-\mathrm{C} 7-\mathrm{N} 1$ & $122.08(17)$ \\
\hline $\mathrm{C} 7-\mathrm{N} 1-\mathrm{H} 1 \mathrm{~A}$ & 115.9 & $\mathrm{O} 1-\mathrm{C} 7-\mathrm{C} 6$ & $121.00(17)$ \\
\hline $\mathrm{C} 8-\mathrm{N} 1-\mathrm{H} 1 \mathrm{~A}$ & 115.9 & $\mathrm{~N} 1-\mathrm{C} 7-\mathrm{C} 6$ & $116.92(16)$ \\
\hline $\mathrm{C} 8-\mathrm{N} 2-\mathrm{C} 9$ & $131.19(16)$ & $\mathrm{C} 10-\mathrm{C} 9-\mathrm{C} 14$ & $119.30(16)$ \\
\hline $\mathrm{C} 8-\mathrm{N} 2-\mathrm{H} 1 \mathrm{~B}$ & 114.4 & $\mathrm{C} 10-\mathrm{C} 9-\mathrm{N} 2$ & $124.87(15)$ \\
\hline $\mathrm{C} 9-\mathrm{N} 2-\mathrm{H} 1 \mathrm{~B}$ & 114.4 & $\mathrm{C} 14-\mathrm{C} 9-\mathrm{N} 2$ & $115.78(16)$ \\
\hline $\mathrm{C} 2-\mathrm{C} 1-\mathrm{C} 6$ & $120.62(17)$ & $\mathrm{N} 2-\mathrm{C} 8-\mathrm{N} 1$ & $114.66(16)$ \\
\hline $\mathrm{C} 2-\mathrm{C} 1-\mathrm{H} 1$ & 119.7 & $\mathrm{~N} 2-\mathrm{C} 8-\mathrm{S} 1$ & $126.92(15)$ \\
\hline $\mathrm{C} 6-\mathrm{C} 1-\mathrm{H} 1$ & 119.7 & $\mathrm{~N} 1-\mathrm{C} 8-\mathrm{S} 1$ & $118.43(13)$ \\
\hline $\mathrm{C} 3-\mathrm{C} 2-\mathrm{C} 1$ & $118.79(17)$ & $\mathrm{C} 11-\mathrm{C} 10-\mathrm{C} 9$ & $119.57(16)$ \\
\hline $\mathrm{C} 3-\mathrm{C} 2-\mathrm{H} 2$ & 120.6 & $\mathrm{C} 11-\mathrm{C} 10-\mathrm{H} 10$ & 120.2 \\
\hline $\mathrm{C} 1-\mathrm{C} 2-\mathrm{H} 2$ & 120.6 & $\mathrm{C} 9-\mathrm{C} 10-\mathrm{H} 10$ & 120.2 \\
\hline $\mathrm{F} 1-\mathrm{C} 3-\mathrm{C} 2$ & $119.46(18)$ & $\mathrm{C} 12-\mathrm{C} 11-\mathrm{C} 10$ & $119.85(16)$ \\
\hline $\mathrm{F} 1-\mathrm{C} 3-\mathrm{C} 4$ & $117.55(18)$ & $\mathrm{C} 12-\mathrm{C} 11-\mathrm{H} 11$ & 120.1 \\
\hline $\mathrm{C} 2-\mathrm{C} 3-\mathrm{C} 4$ & $122.98(18)$ & $\mathrm{C} 10-\mathrm{C} 11-\mathrm{H} 11$ & 120.1 \\
\hline $\mathrm{C} 5-\mathrm{C} 4-\mathrm{C} 3$ & $117.70(18)$ & $\mathrm{C} 13-\mathrm{C} 12-\mathrm{C} 11$ & $121.42(16)$ \\
\hline $\mathrm{C} 5-\mathrm{C} 4-\mathrm{H} 4$ & 121.1 & $\mathrm{C} 13-\mathrm{C} 12-\mathrm{Br} 1$ & $119.31(13)$ \\
\hline $\mathrm{C} 3-\mathrm{C} 4-\mathrm{H} 4$ & 121.1 & $\mathrm{C} 11-\mathrm{C} 12-\mathrm{Br} 1$ & $119.27(13)$ \\
\hline $\mathrm{C} 4-\mathrm{C} 5-\mathrm{C} 6$ & $120.91(18)$ & $\mathrm{C} 14-\mathrm{C} 13-\mathrm{C} 12$ & $118.76(16)$ \\
\hline $\mathrm{C} 4-\mathrm{C} 5-\mathrm{H} 5$ & 119.5 & $\mathrm{C} 14-\mathrm{C} 13-\mathrm{H} 13$ & 120.6 \\
\hline $\mathrm{C} 6-\mathrm{C} 5-\mathrm{H} 5$ & 119.5 & $\mathrm{C} 12-\mathrm{C} 13-\mathrm{H} 13$ & 120.6 \\
\hline $\mathrm{C} 1-\mathrm{C} 6-\mathrm{C} 5$ & $118.97(17)$ & $\mathrm{C} 13-\mathrm{C} 14-\mathrm{C} 9$ & $121.10(17)$ \\
\hline $\mathrm{C} 1-\mathrm{C} 6-\mathrm{C} 7$ & $123.33(17)$ & $\mathrm{C} 13-\mathrm{C} 14-\mathrm{H} 14$ & 119.5 \\
\hline $\mathrm{C} 5-\mathrm{C} 6-\mathrm{C} 7$ & $117.62(16)$ & $\mathrm{C} 9-\mathrm{C} 14-\mathrm{H} 14$ & 119.5 \\
\hline $\mathrm{C} 6-\mathrm{C} 1-\mathrm{C} 2-\mathrm{C} 3$ & $-0.1(3)$ & $\mathrm{C} 8-\mathrm{N} 2-\mathrm{C} 9-\mathrm{C} 10$ & $-28.7(3)$ \\
\hline $\mathrm{C} 1-\mathrm{C} 2-\mathrm{C} 3-\mathrm{F} 1$ & $179.66(16)$ & $\mathrm{C} 8-\mathrm{N} 2-\mathrm{C} 9-\mathrm{C} 14$ & $154.04(18)$ \\
\hline $\mathrm{C} 1-\mathrm{C} 2-\mathrm{C} 3-\mathrm{C} 4$ & $-1.4(3)$ & $\mathrm{C} 9-\mathrm{N} 2-\mathrm{C} 8-\mathrm{N} 1$ & $-175.93(16)$ \\
\hline $\mathrm{F} 1-\mathrm{C} 3-\mathrm{C} 4-\mathrm{C} 5$ & $-179.80(16)$ & $\mathrm{C} 9-\mathrm{N} 2-\mathrm{C} 8-\mathrm{S} 1$ & $3.8(3)$ \\
\hline $\mathrm{C} 2-\mathrm{C} 3-\mathrm{C} 4-\mathrm{C} 5$ & $1.2(3)$ & $\mathrm{C} 7-\mathrm{N} 1-\mathrm{C} 8-\mathrm{N} 2$ & $6.3(3)$ \\
\hline $\mathrm{C} 3-\mathrm{C} 4-\mathrm{C} 5-\mathrm{C} 6$ & $0.4(3)$ & $\mathrm{C} 7-\mathrm{N} 1-\mathrm{C} 8-\mathrm{S} 1$ & $-173.53(14)$ \\
\hline $\mathrm{C} 2-\mathrm{C} 1-\mathrm{C} 6-\mathrm{C} 5$ & $1.6(3)$ & $\mathrm{C} 14-\mathrm{C} 9-\mathrm{C} 10-\mathrm{C} 11$ & $-1.1(3)$ \\
\hline $\mathrm{C} 2-\mathrm{C} 1-\mathrm{C} 6-\mathrm{C} 7$ & $178.38(16)$ & $\mathrm{N} 2-\mathrm{C} 9-\mathrm{C} 10-\mathrm{C} 11$ & $-178.32(16)$ \\
\hline $\mathrm{C} 4-\mathrm{C} 5-\mathrm{C} 6-\mathrm{C} 1$ & $-1.8(3)$ & $\mathrm{C} 9-\mathrm{C} 10-\mathrm{C} 11-\mathrm{C} 12$ & $0.5(3)$ \\
\hline $\mathrm{C} 4-\mathrm{C} 5-\mathrm{C} 6-\mathrm{C} 7$ & $-178.72(16)$ & $\mathrm{C} 10-\mathrm{C} 11-\mathrm{C} 12-\mathrm{C} 13$ & $0.4(3)$ \\
\hline $\mathrm{C} 8-\mathrm{N} 1-\mathrm{C} 7-\mathrm{O} 1$ & $3.4(3)$ & $\mathrm{C} 10-\mathrm{C} 11-\mathrm{C} 12-\mathrm{Br} 1$ & $179.54(13)$ \\
\hline $\mathrm{C} 8-\mathrm{N} 1-\mathrm{C} 7-\mathrm{C} 6$ & $-176.01(16)$ & $\mathrm{C} 11-\mathrm{C} 12-\mathrm{C} 13-\mathrm{C} 14$ & $-0.6(3)$ \\
\hline $\mathrm{C} 1-\mathrm{C} 6-\mathrm{C} 7-\mathrm{O} 1$ & $-154.97(17)$ & $\mathrm{Br} 1-\mathrm{C} 12-\mathrm{C} 13-\mathrm{C} 14$ & $-179.76(13)$ \\
\hline $\mathrm{C} 5-\mathrm{C} 6-\mathrm{C} 7-\mathrm{O} 1$ & $21.9(3)$ & $\mathrm{C} 12-\mathrm{C} 13-\mathrm{C} 14-\mathrm{C} 9$ & $0.0(3)$ \\
\hline $\mathrm{C} 1-\mathrm{C} 6-\mathrm{C} 7-\mathrm{N} 1$ & $24.4(2)$ & $\mathrm{C} 10-\mathrm{C} 9-\mathrm{C} 14-\mathrm{C} 13$ & $0.9(3)$ \\
\hline
\end{tabular}


supporting information

$\mathrm{C} 5-\mathrm{C} 6-\mathrm{C} 7-\mathrm{N} 1 \quad-158.75(16) \quad \mathrm{N} 2-\mathrm{C} 9-\mathrm{C} 14-\mathrm{C} 13 \quad 178.36(14)$

Hydrogen-bond geometry $\left(\AA,{ }^{\circ}\right)$

\begin{tabular}{lllll}
\hline$D-\mathrm{H} \cdots A$ & $D-\mathrm{H}$ & $\mathrm{H} \cdots A$ & $D \cdots A$ & $D-\mathrm{H} \cdots A$ \\
\hline $\mathrm{N} 1-\mathrm{H} 1 A \cdots \mathrm{S} 1^{\mathrm{i}}$ & 0.88 & 2.69 & $3.5081(15)$ & 154 \\
$\mathrm{~N} 2-\mathrm{H} 1 B^{\cdots} \mathrm{O} 1$ & 0.88 & 1.88 & $2.610(2)$ & 139 \\
$\mathrm{C} 10-\mathrm{H} 10 \cdots \mathrm{S} 1$ & 0.95 & 2.65 & $3.2319(18)$ & 120 \\
$\mathrm{C} 1-\mathrm{H} 1 \cdots \mathrm{S}{ }^{\mathrm{ii}}$ & 0.95 & 2.81 & $3.7312(18)$ & 165 \\
\hline
\end{tabular}

Symmetry codes: (i) $-x+1,-y+1,-z$; (ii) $-x,-y+1,-z$. 